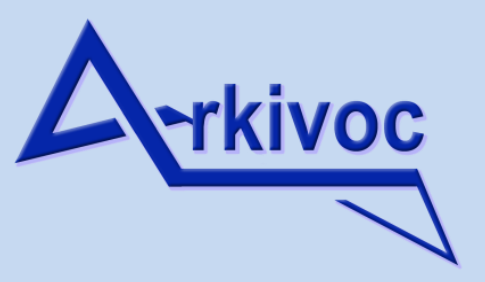

Free to Authors and Readers
A Platinum Open Access Journal for Organic Chemistry
Review

Arkivoc 2021, part iv, 89-104

\title{
Identifying bioactivity of pseudo-natural products using the Cell Painting assay
}

\author{
José-Manuel Gally, ${ }^{\text {a }}$ Axel Pahl, ${ }^{\text {b }}$ and Herbert Waldmann*a,c \\ ${ }^{a}$ Department of Chemical Biology, Max-Planck-Institute of Molecular Physiology, \\ Otto-Hahn-Straße 11, 44227 Dortmund, Germany \\ ${ }^{b}$ Compound Management and Screening Center, Dortmund \\ Otto-Hahn-Str. 11, 44227 Dortmund (Germany) \\ ${ }^{c}$ Faculty of Chemistry and Chemical Biology, Technical University Dortmund, \\ Otto-Hahn-Straße 6, 44227 Dortmund, Germany \\ Email: herbert.waldmann@mpi-dortmund.mpg.de
}

\section{Dedicated to Prof. Horst Kunz on the occasion of his $80^{\text {th }}$ anniversary}

Received 08-31-2020

Accepted 09-18-2020

Published on line 09-25-2020

\begin{abstract}
Natural Products (NP) are a major source of inspiration to develop novel bioactive substances. Various strategies were developed to make use of the relevance of NPs in drug design strategies. It has been shown that NP-derived fragments may still be of biological relevance. Thus, combining various different NP-derived fragments may result in new biologically relevant molecules. These compounds retain some physicochemical properties of NPs but are not accessible through biosynthesis and were therefore termed pseudo-NPs. Since it is not possible to infer the bioactivity of pseudo-NPs from their NP-derived fragments, it is not straightforward to identify their potential targets. Hence more general, morphological phenotypic screens may be the best methods to identify impacted pathways. We highlight the potential of the pseudo-NP approach combined with a recent morphological-, image-based screening technology termed Cell Painting.
\end{abstract}

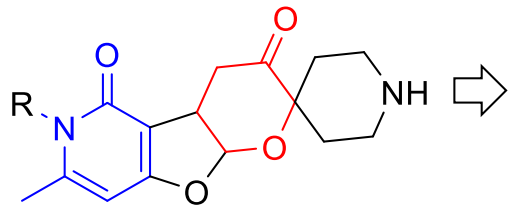

Pseudo-Natural Product
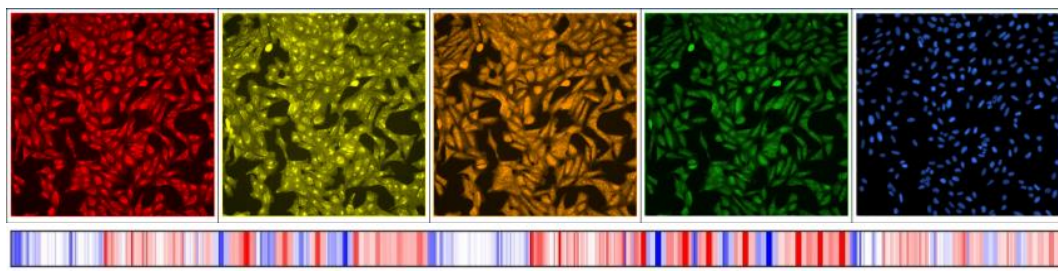

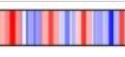

Cell Painting Assay

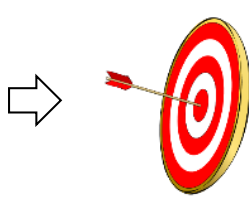

Target Hypothesis

Keywords: Pseudo-natural product, cell painting, biological target, biological activity 


\section{Table of Contents}

1. Introduction

2. Pseudo-natural Products

3. Challenges in Finding Bioactivity

3.1 Target-based in vitro

3.2 Morphological image-based analysis

3.3 Target-agnostic high content screening assays, Cell Painting (CP)

4. Using Cell Painting to Identify Targets and Mode of Action of Pseudo-natural Products

4.1 Pyrano-furo-pyridones

4.2 Indocinchona alkaloids

4.3 Autoquin

5. Conclusions

References

\section{Introduction}

Natural Products (NPs) have been a major source of inspiration in drug discovery pipelines for decades. Cragg et al. showed that between January 1981 and September 2019, no less than $60.4 \%$ of new approved drugs are either genuine NPs, NP-derived compounds or NP-mimics (synthetic compounds that mimic NP pharmacophores). ${ }^{1}$ Indeed, a very long process of evolution developed NPs to interact with specific targets, making them biologically prevalidated molecules. ${ }^{2}$ Several strategies have been developed to convert existing NPs into synthetic compounds with enhanced physicochemical properties. A common approach is to only change relatively small parts of the molecule, so that in many cases the modified NPs still have bioactivities that are very close to the parent NP.

The so-called Biology-oriented Synthesis (BIOS) strategy, developed in the Waldmann group ${ }^{3}$, pushes the idea of transferable bioactivity further by reducing NPs to their core scaffolds and even to NP fragments. A chemoinformatics analysis not only indicated that NP scaffolds could retain some of the parent NPs bioactivities, but more than half of the resulting representative compounds for fragment classes were readily purchasable. ${ }^{4}$ This is especially valuable because the availability of the NP source material can be a limiting factor. As an indication, Kirchmair et al. estimated that on average only $10 \%$ of NPs are readily purchasable. ${ }^{5}$

It is however sometimes desirable to explore novel biological space by overcoming the parent NPs known bioactivities. A strategy of ring distortion/modification was developed by Hergenrother and co-workers, ${ }^{6}$ that consists in disrupting the ring systems of NPs scaffolds. Modifications are carefully performed through appropriate synthetic steps, to mimic enzymatic processes in intermediate metabolite synthesis. The resulting NP-derived molecules resemble NPs in the sense that they are still stereochemically complex. Since a large number of new compounds can be obtained through this method, exploring thereby new chemical space, it is commonly referred to as "Complexity to Diversity" (CtD). ${ }^{6}$

NPs occupy a broad yet delimited chemical space, ${ }^{5}$ which can merely be considered a drop in the ocean when compared to the estimated $10^{60}$ possible different drug-like molecules. ${ }^{7}$ In order to efficiently navigate novel chemical space, approaches such as Fragment-Based Drug Design (FBDD) have been developed and yielded convincing results. ${ }^{8,9}$ The combination of biologically-relevant NP fragments to form new NP-like scaffolds and thus unlock novel chemical space, not explored by Nature, is hence promising. The resulting 
compounds resemble NPs but are not obtainable through biosynthesis and were therefore termed pseudoNatural Products (pseudo-NPs). ${ }^{10,11}$ This term was also previously employed by Suga et al. to describe circular peptides ${ }^{12,13}$ and Oshima et al. to describe products from intercepted biosynthetic pathways. ${ }^{14,15}$

\section{Pseudo-natural Products}

A few principles have been taken into account in the development of the pseudo-NP approach. ${ }^{10}$ First, chirality and thus shape is a defining property for NPs and their implication in specific and selective biological processes. Pseudo-NPs may also reach such structural complexity through appropriate synthetic pathways. Second, an appropriate balance of heteroatoms in the resulting scaffold could be aimed for by combining fragments either rich in nitrogen or oxygen atoms, respectively more predominant in synthetic and natural compounds. Third, combining NP fragments from NPs with distinct bioactivities may help to maximize the discovery rate of novel bioactivity. Finally, combining NP fragments that are biosynthetically unrelated may facilitate the binding to different targets of distinctive pathways, which may result in unprecedented bioactivities.

To further increase the number of possibilities, NP fragments could be associated with different linkage types (Figure 1). Hence the connection could be either direct, such as spiro, edge and bridged fusions, or indirect such as monopodal and bipodal connections. In addition, it is possible to vary the connection positions of the NP fragments to maximize the number of possibilities.

\section{Challenges in Finding Bioactivity}

Different technologies can be employed for the identification of biological activity of small molecules, which have strengths and weaknesses based on the employed assay principles.

\subsection{Target-based in vitro}

Since the resulting bioactivity of pseudo-NPs cannot be inferred from the initial NP fragments alone, identifying the target(s) for a pseudo-NP constitutes a challenge. Numerous different bioassays have been developed and optimized for specific targets. ${ }^{16}$ Isolated target-based assays are artificial systems where the alteration of the activity of a specific, predefined target by a small molecule can be monitored over time. The success of this technique mostly depends on the availability of the target and a detection method with sufficient sensibility. In most cases target-based assays remain relatively straightforward to set up and automate for larger scale analysis. ${ }^{17}$

Biochemical assays evaluate the binding of a small molecule to the hypothesized isolated target of interest, which can be for instance a protein or a complex of proteins in the case of Protein-Protein interactions (PPi). This type of assays has the advantage of fine tuning and greater control over the experimental conditions, including buffer composition, $\mathrm{pH}$, temperature, ionic concentration and reaction volume. ${ }^{18}$ It is however adapted for only one target at a time, and conditions have to be optimized to detect the correct type of affinity (agonist, antagonist, etc.) as well. This may result in identification of compounds that would otherwise be found active against other targets. Target-based assays include a variety of biochemical experiments, ${ }^{16}$ such as fluorescence-based assays (i.e. fluorescence quenching, ${ }^{19}$ fluorescence polarization, ${ }^{20}$ etc.), radioligand-binding ${ }^{21}$ and coupled-enzyme assays (i.e. for ATP/ADP measurements ${ }^{22}$ ). 
Connection Type Pseudo-NP example

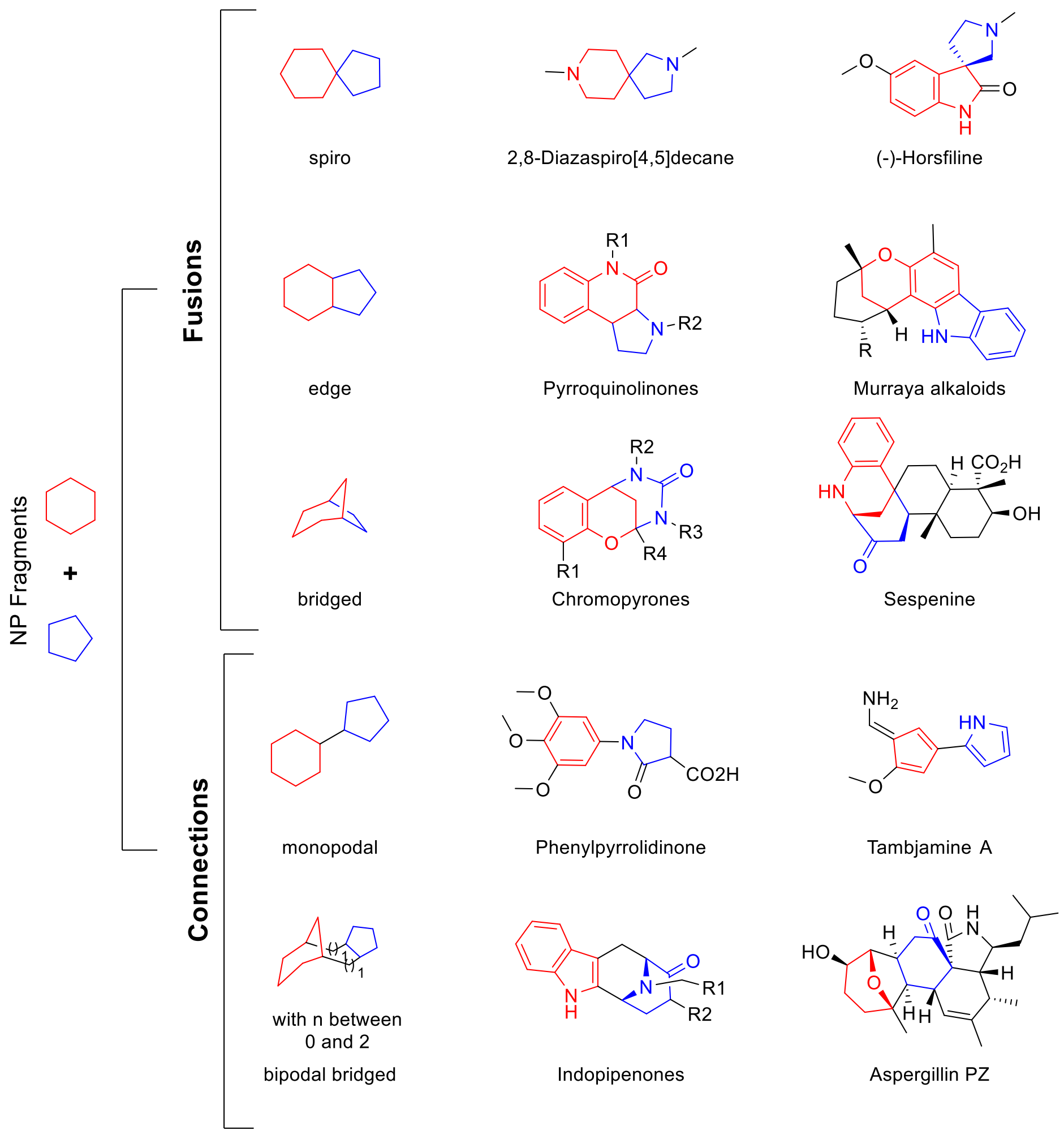

Figure 1. Examples of different NP-derived fragments combinations in pseudo-NPs and NPs. Adapted from ref. 10. 
Cell-based assays are another type of extensively investigated target-based biological assay. ${ }^{23}$ They do not focus on the binding of a probed small molecule with a target but rather investigate target function. In such systems the target is not isolated but rather overexpressed in a cell. This allows the investigation of more categories of targets such as GPCRs (G-protein coupled receptors). ${ }^{17}$ Since the overexpression of wild-type genes can lead to mutant phenotypes, it has been exploited by geneticists as a complementary approach to loss of function screens. Examples of applications include functional assays measuring second messengers ${ }^{24}$ (e.g. cAMP and $\mathrm{Ca}^{2+}$ ), ion flux, ${ }^{25}$ transport to or from vesicles. ${ }^{26}$ Cell-based assays have a broader window for target identification than biochemical assays because they highlight the effect of the probed small molecule on a pathway rather than on a single target. The analysis of such assays is not as straightforward as with biochemical assays and follow-up experiments are required to validate the hypothesized mode of action of the effector. ${ }^{27}$ The chance of missing potential bioactivity remains however high as only one pathway is probed at a time.

\subsection{Morphological image-based analysis}

In cell assays with image-based readout morphological changes in the cell induced by the modulation of a biological pathway of interest are coupled to a machine-readable output, e.g. the localization or distribution of fluorescence, which can be recorded by a microscope and analysed using suitable software.

This assay principle was successfully used for investigation of autophagy, ${ }^{28}$ a highly regulated homeostatic process where cells degrade their internal damaged or superfluous components. The degraded products may get recycled and serve as an alternative energy source during phases of metabolic stress. Autophagy is associated with several diverse diseases, among them cancer. ${ }^{29,30}$ The assay is performed in cell lines that are stably expressing EGFP-LC3 (enhanced green fluorescent protein tagged light chain 3; the LC3 system is a biomarker for autophagy ${ }^{31}$ ). The cells are then incubated with the test compounds and simultaneously submitted to stress conditions to induce autophagy. This can be achieved either by starvation through the medium or by inhibition of mTOR (mammalian target of rapamycin) using rapamycin.

In the final image analysis, the granular accumulation of the fluorescent-tagged LC3 on autophagosomes can be detected and quantified (Figure 2).
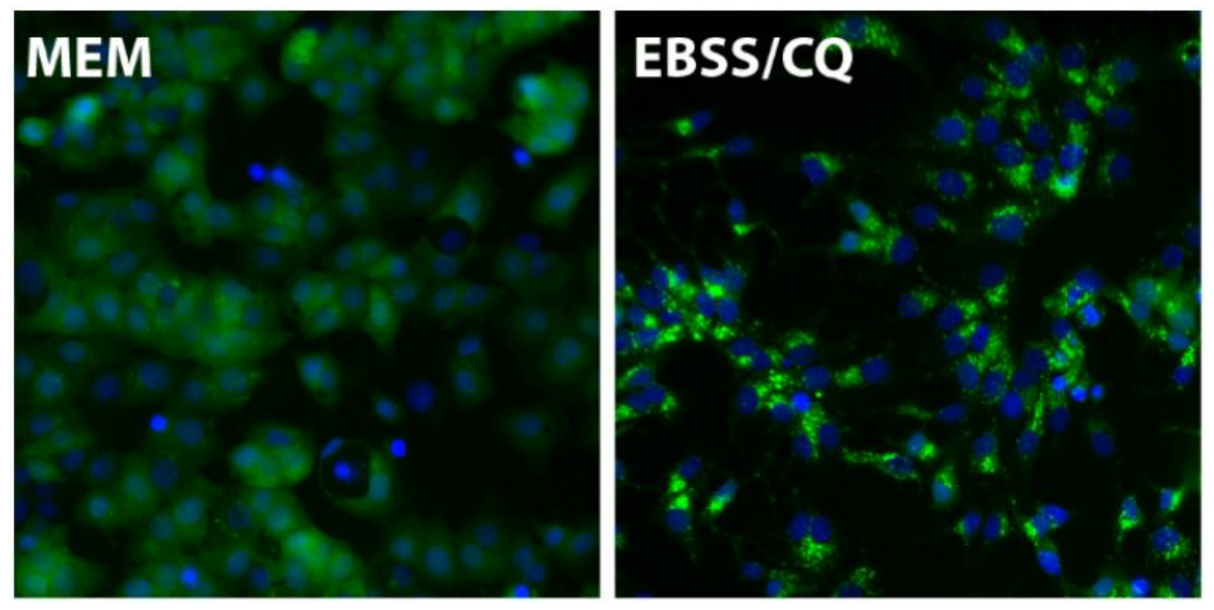

Figure 2. Fed control (left) vs. starvation-induced autophagy (right); MEM: Minimum Essential Medium, EBSS: Earle's Balanced Salt Solution, CQ: Chloroquine. 
In living cell-based systems, the target pathway of the assay is embedded in the cellular machinery. A test compound has to achieve sufficient bioavailability before it can interact with the target pathway. The compound has to enter through the outer cell membrane (either passively by diffusion or by active transport), and needs to have chemical stability in the cytoplasm over the time of the measurement. Because of this increased complexity, cellular assays are often considered to be more physiologically relevant than in vitro biochemical assays that use isolated proteins in artificial cell-free buffers.

Since morphological assays often target complete pathways of high biological relevance instead of single targets, they have an increased likelihood of identifying compounds with interesting activity. On the other hand, further investigations are necessary to elucidate the exact target of a compound once an initial activity in a pathway has been found.

Morphological assays are more difficult to set up (e.g. they usually employ transfected cell lines) and are more demanding to adapt for higher-throughput automated testing which requires expensive instrumentation for the recording and analysis of the readout.

Finally, since the readout focuses on the modulation of one specific pathway in the cell, there is still a high risk of missing activity of a compound.

\subsection{Target-agnostic high content screening assays, Cell Painting (CP)}

Considering the advantages and disadvantages of the methods for finding activity reveals that a powerful solution for detecting activity of new compounds would be a completely target-agnostic unbiased assay that can detect a multitude of different activities without focus on specific targets or pathways. Ideally, that method would also be suitable for automation and be capable of medium to high throughput analysis.

The Cell Painting assay, a multiplexed morphological screening technique developed at the Broad institute by the Carpenter group, ${ }^{32}$ comes very close to this definition. Whereas it has found application in a number of fields, among them gene expression profiling, ${ }^{33}$ exploring options for drug repurposing, ${ }^{34}$ and assessing and optimizing diversity of bioactivity, ${ }^{35,36}$ the assay principle is described below in the context of identifying activity in small molecules.

Cells of a suitable cell line (U2OS and A549 are common examples) are incubated with test compounds and DMSO controls ( 24 or $48 \mathrm{~h}$ ) in microtiter plates (384 format). The cells are then fixed and simultaneously stained with up to 6 different dyes which selectively stain the following compartments of the cell: nucleus, endoplasmic reticulum, nucleoli / cytoplasmic RNA, F-actin cytoskeleton / golgi / plasma membrane and mitochondria.

The dyes have fluorescent responses at 5 different wavelengths that are recorded separately by the microscope (two dyes have overlapping wavelengths), which takes up to 9 images per each microtiter well. The resulting set of 17,280 images per plate is then analyzed with the Open Source software CellProfiler, also developed by the Carpenter group. ${ }^{37}$ CellProfiler identifies the cells in the images and extracts hundreds of morphological features for each cell from the stained compartments. After statistical aggregation to each test compound and normalization to the DMSO controls these features are collected into profiles which describe the morphological changes that each test compound has induced in the cells. In addition to the feature profiles an induction value can be calculated which describes the percentage of features in the full profile that are significantly changed compared to the controls.

Every plate contains two columns of controls, leaving room for 352 test compounds. When the assay is performed with replicates (at least triplicates are recommended) the number of images increases accordingly (51,840 images for one triplicate measurement of 352 compounds). The analysis of this high number of images 
requires significant computing capacities. It can not be processed on a desktop machine anymore and is usually performed on computing clusters.

The generated biological feature profiles of the tested compounds can be compared to each other or to a set of reference compounds with known biological activity. Since the similarity of the profiles can be calculated (e.g. using Pearson similarity), it is possible to determine a list of reference compounds whose biological profiles are most similar to a given test compound. The annotated information of these similar reference compounds can be used to derive a hypothesis about the target or the pathway perturbed by the test compound (Figure 3).

\section{a}

Cell Painting assay

in 384 well format

in triplicates

(352 compounds/plate

\& 32 DMSO controls)

9 microscope
sites per well

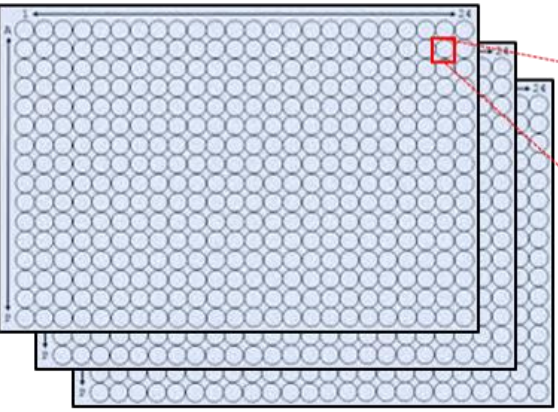

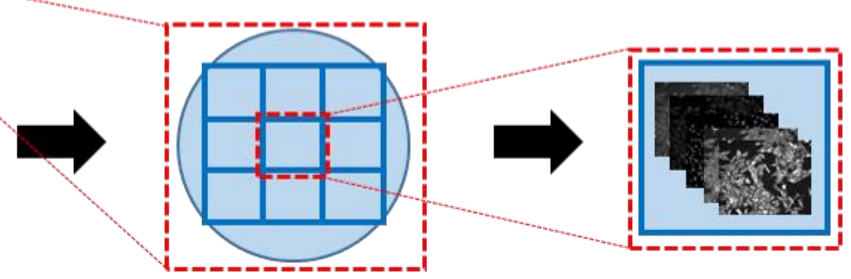

b

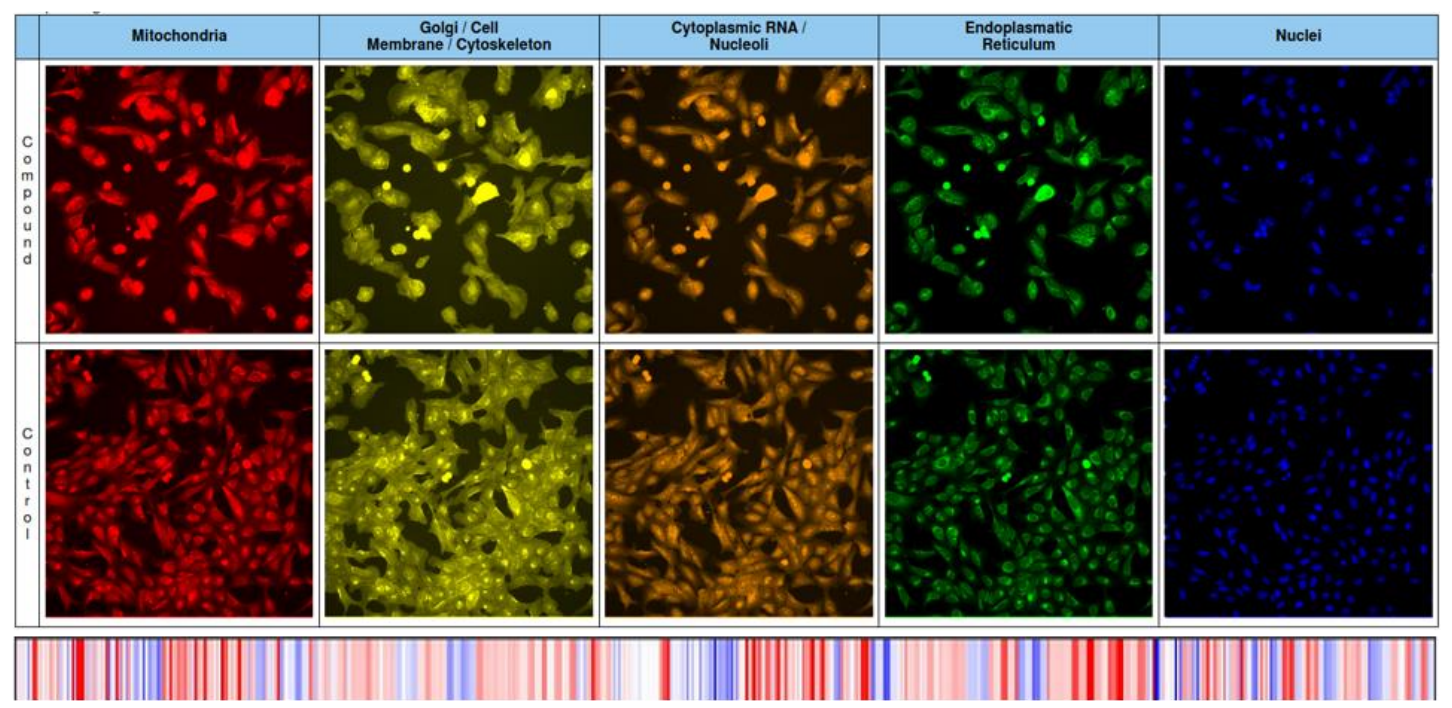

C

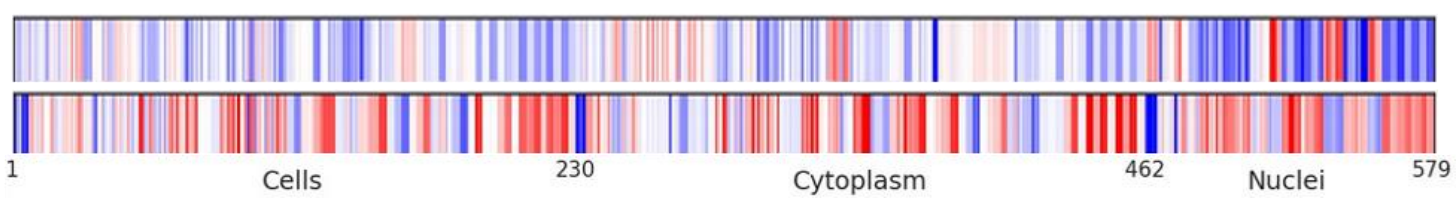

Figure 3. Principle of the Cell Painting assay. a) Assay setup and image acquisition. b) Example images of treated and control cells and the resulting phenotypic feature profile for the tubulin inhibitor Docetaxel. c) Cell Painting profiles of mTOR inhibitor Everolismus and Aurora inhibitor Hesperadin. Color scale ranges from -15 (blue) to +15 (red) for all examples. 
The greatest advantage of the Cell Painting assay is the unbiased detection of biological activity of a test compound. It does not require a prior target hypothesis and will pick up any morphological changes that are induced in the treated cells and differ from the controls. From all methods described above, Cell Painting has the highest likelihood of finding activity for new, hitherto unknown compounds.

Since the assay does not require any modifications of the cell line (e.g. no overexpression of a target or pathway, no internalization of markers) during the compound incubation time, it has a high physiological relevance within the given limits of a cellular assay. Like all cell-based assays it requires the compounds to be bio-available in the cells and to be chemically stable over the time of incubation. Because Cell Painting relies on changes in the cell morphology, only targets whose modulation induce morphological changes can be detected. The assay is automatable to medium throughput but the analysis is computationally intensive and requires dedicated hardware.

Cell Painting often cannot directly provide the target of a tested compound, it rather gives a "high level" information about a modulated pathway. The quality of these pathway hypotheses that were generated by comparing the biological profiles of a test compound to those of reference compounds strongly depends on the set of reference compounds, its size and target diversity, as well as the quality of their target annotations. Because of the unbiased nature of the assay, it will not only detect highly interesting and relevant biological activity, but also activity of lower interest as well as toxicity. Finally, for the elucidation of a concrete target, follow-up experiments are necessary.

\section{Using Cell Painting to Identify Targets and Mode of Action of Pseudo-natural Products}

\subsection{Pyrano-furo-pyridones}

Christoforow et al. ${ }^{38}$ describe the synthesis of pyrano-furo-pyridones (PFPs), a class of pseudo-NPs formed by the combination of the NP fragments pyridone and dihydropyran in three isomeric arrangements (Figure 4a).

From the results of the Cell Painting assay two compounds of one structural class with high induction values were selected for in-depth target elucidation (Figure 4b: PFP-1 and PFP-2).

The target annotations of the identified references with high biological similarity to the synthesized compounds indicated activity in several pathways, which were subsequently investigated in more detail: mitochondrial respiration, autophagy, glucose uptake inhibition and Wnt / Hedgehog (Hh) signaling. In followup cell-based assays autophagy and Hh signaling as well as glucose uptake could be excluded as potential modes of action. A high biological profile similarity of the synthesized pseudo-NPs to Aumitin (Figure 4c), a known inhibitor of mitochondrial respiration which targets the mitochondrial complex l, was observed. This pathway is also connected to the regulation of reactive oxygen species (ROS), which is involved in aging and other diseases. It has recently gained interest in cancer research, because the formation of ROS might selectively enhance the cytotoxicity to cancerous cells thereby limiting cancer progression. ${ }^{39}$ By conducting further studies the induction of ROS and the inhibition of mitochondrial complex I could indeed be confirmed as mechanisms of action for the synthesized compounds.

This example shows the value of the Cell Painting assay in formulating target hypotheses for compounds with unprecedented activity. Here, it was the basis for the identification of a mode of action for a class of pseudo-NPs, which are structurally unrelated to the known inhibitors. The study also highlights the necessity for follow-up studies to further elucidate the formulated target hypotheses. 
a<smiles>[R]N1CCCC2=C(O)C(=O)[O+]=C21</smiles>

Functionalized pyridone precursor

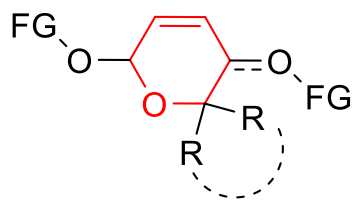

Functionalized dihydropyran precursor

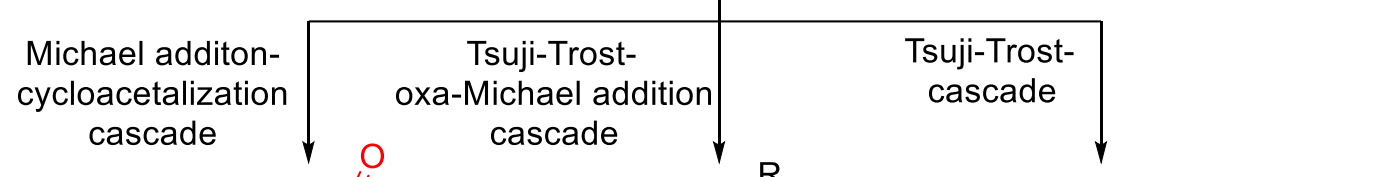

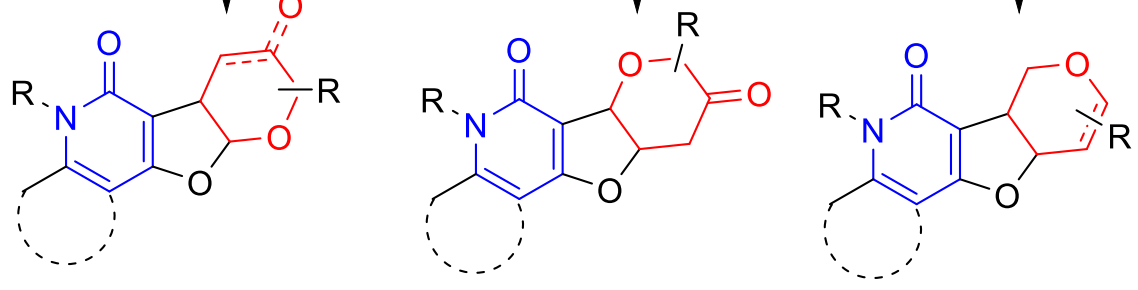

Pyrano-furo-pyridone pseudo-NPs

b<smiles>COc1ccc2[nH]cc(CCn3c(C)cc4c(c3=O)C3CC(=O)C5(CCN(C(C)(C)C)CC5)OC3O4)c2c1</smiles>

C<smiles>C=C/C=C/c1cc(C)nc(Nc2ccc(NC(=O)c3ccccc3Cl)cc2)n1</smiles>

Figure 4. a) Design principle of pyrano-furo-pyridones (PFP). b) Structures of PFP-1 and PFP-2, compounds chosen for in-depth biological characterization. c) Structure of Aumitin. Adapted from ref. 38.

\subsection{Indocinchona alkaloids}

Another example where Cell Painting supported the target elucidation process was reported by Foley et al. ${ }^{40}$ In their pseudo-NPs the cinchona alkaloid scaffold is combined with indoles and aza-indoles, forming previously undescribed indocinchona alkaloids (Figure 5).

Especially the combination with aza-indoles - yielding the novel class of azaquindoles - showed interesting activity. The Cell Painting feature profiles showed similarity to the VPS34 inhibitor SAR405 (Figure 5d) and the $\beta$-adrenergic receptor agonist terbutaline. These two target hypotheses were investigated by secondary assays, which quickly ruled out the $\beta$-adrenergic receptor activity. VPS34 plays an important role in the autophagy process and is involved in the autophagosome biogenesis downstream of mTOR. It therefore fits the VPS34 target hypothesis that Azaquindole-1 (Figure 5c) inhibited starvation- and rapamycin-induced autophagy with high efficacy $\left(\mathrm{IC}_{50}: 0.04 \mu \mathrm{M}\right.$ and $0.1 \mu \mathrm{M}$ respectively) and a potent inhibition of VPS34 (IC 50 : $0.35 \mu \mathrm{M})$ could finally be confirmed in an in vitro kinase panel assay. 
a

Cinchona alkaloids Indole alkaloids

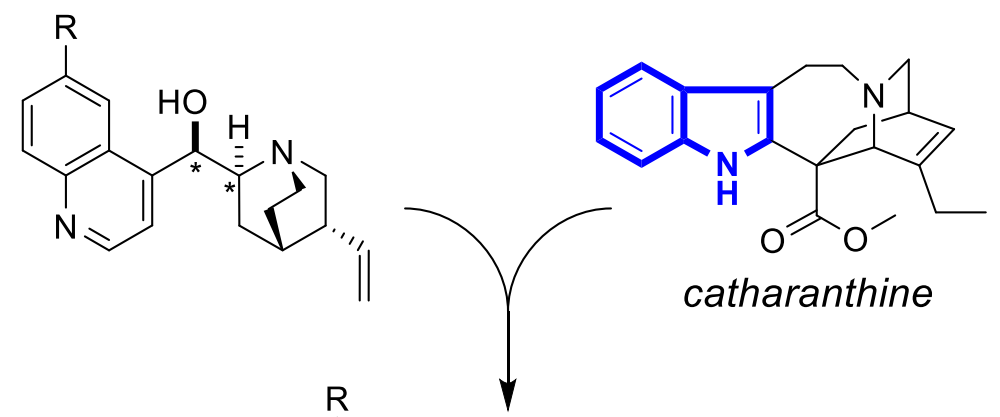<smiles>[Y]c1ccc2nccc(C(O)[C@H]3CC4CCN3c3c4[nH]c4ccccc34)c2c1</smiles>

indocinchona pseudo-NPs

b<smiles>[R]c1ccc2nccc([C@@H](O)[C@H]3CC4CCN3CC4=O)c2c1</smiles>

ketones from: quinidine, quinine

c<smiles>COc1ccc2nccc([C@@H](O)[C@H]3CC4CCN3c3c4[nH]c4nc(C)c(Br)cc34)c2c1</smiles>

Azaquindole-1

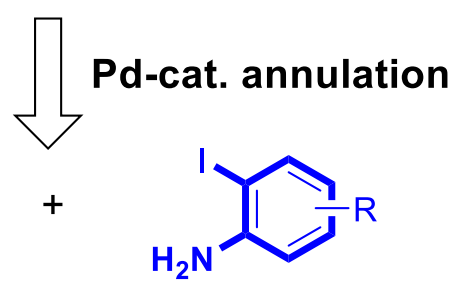

2-iodoanilines

(commercially available)

d

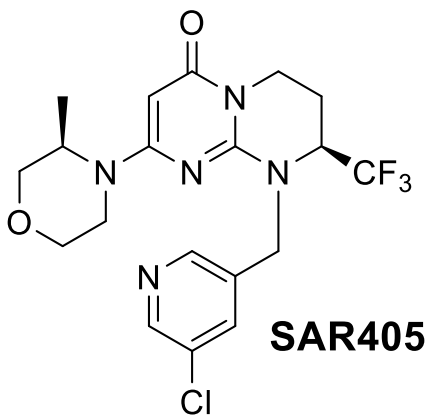

Figure 5. Indocinchona pseudo-NPs. a) Design principle. b) Synthetic approach. c) Structure of Azaquindole-1. d) Structure of SAR405. Adapted from ref. 40.

The paper shows how Cell Painting together with the autophagy assay as another morphological cellbased technique enabled the target elucidation of the novel pseudo-NP compound class of azaquindoles, where the final target could be confirmed by an in vitro assay. Remarkably, the identified activity on VPS34 is not shared by either of the two parent scaffolds emphasizing the potential of the pseudo-NP approach to generate new compounds with unprecedented activity.

\subsection{Autoquin}

Another class of cinchona alkaloid-inspired natural product derivatives, the so-called autoquins, was developed by Laraia et al. ${ }^{41}$ Although these compounds are no pseudo-NPs by definition (they are not formed 
by the combination of multiple natural product fragments, but rather by the modification of the NP itself), they are new chemical entities with unknown biological activity and the same principles for target elucidation apply. The autoquins were designed as a more versatile and flexible structural class than their related derivatives, the oxautins, ${ }^{42}$ which are accessible by an intramolecular cyclization (Figure 6).

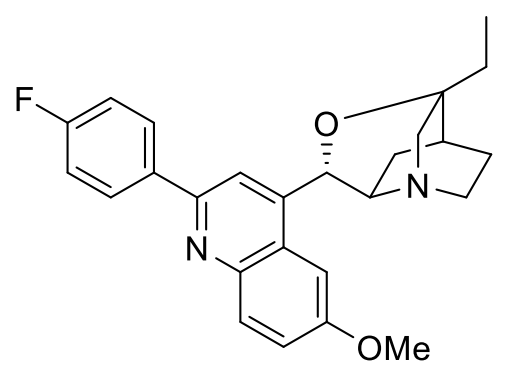

Oxautin-1

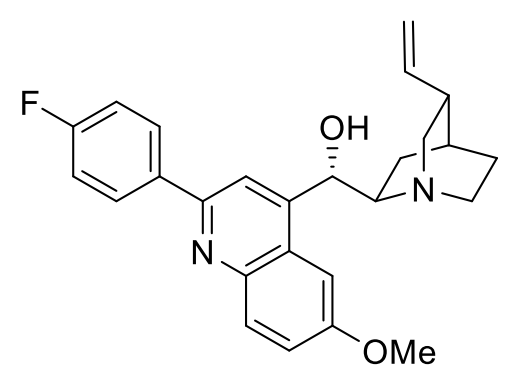

Autoquin

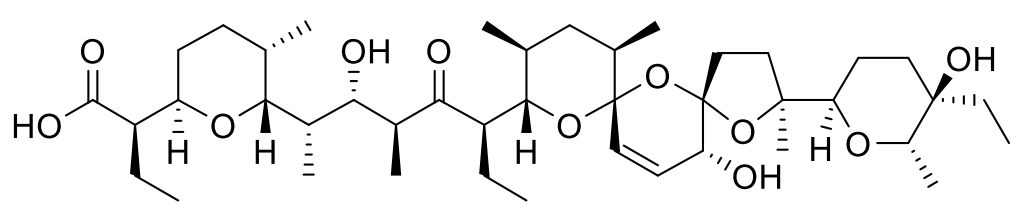

Salinomycin

Figure 6. Structures of Oxautin-1, Autoquin and Salinomycin.

Like the oxautins, Autoquin (Figure 6) was identified as an autophagy inhibitor, with a high activity in starvation-induced autophagy (IC $\left.\mathrm{C}_{50}: 0.56 \mu \mathrm{M}\right)$.

Initially, it was attempted to identify the target by compound-centric chemical proteomics (CCCP), ${ }^{43,44}$ a technique where the molecule in question is immobilized (e.g. on magnetic beads) via a spacer. The beads are then treated with cell lysates and through binding of the molecule to its target an enrichment of the target on the beads is achieved and can be characterized. A drawback of this method is the attachment of the required spacer to the molecule in question. The spacer needs to have the right length and it has to be attached at the right position in the molecule, so that the activity is retained. Moreover, the preparation of the molecule with the spacer requires extra synthetic steps and might be challenging. The pull-down experiments with the probe derivative of Autoquin were performed on cell lysates and identified one potential target, ferrochelatase (FECH) which is located in the mitochondria and catalyzes the insertion of iron in the last step of the heme biosynthesis. Since there is no direct link reported between $\mathrm{FECH}$ and autophagy, it was attempted to validate FECH as a target. To that regard, a competition assay was performed as a follow-up, where the pull-down experiments were repeated in the presence of soluble unchanged Autoquin. As there was no change in the enrichment in the presence of the competition inhibitor and Autoquin was also not able to stabilize $\mathrm{FECH}$ against thermal denaturation, FECH was finally ruled out as a direct target of Autoquin.

Cell Painting identified several reference compounds with high biological similarity to Autoquin, among them Perphenazine (a non-selective GPCR ligand), Loperamide (an opioid receptor agonist) and Toremifene (an estrogen receptor ligand). Although the three compounds are structurally unrelated and share no obvious biological target, they all are reported to be lysosomotropic compounds.

Lysosomes are spherical vesicles within the cell that contain hydrolytic enzymes for the breakdown of large biomolecules, including proteins and peptides. They are separated from the cytoplasm by an intracellular 
lipid bilayer. Lysosomotropic compounds typically carry at least one basic nitrogen and are lipophilic, which enables them to pass the intracellular membrane into the lysosome. Because of the low $\mathrm{pH}$ of $\sim 4.5-5$ of this compartment, the compounds get protonated and become trapped inside. ${ }^{45}$ The lysosomotropic activity of Autoquin could indeed be confirmed by a phenotypic assay using Lysotracker Red as the staining reagent.

The collective activity findings from the pull-down experiments and the Cell Painting assay for this compound pointed towards the reported bioactivity of the natural product Salinomycin (Figure 6), which sequesters iron to the lysosomes, inhibiting autophagy and causing ferroptosis. It was therefore speculated that the finding of ferrochelatase as a pull-down hit was due to iron chelation and not target-specific. This was experimentally supported by the findings that Autoquin significantly increased lysosomal mass, lysosomal Fe ${ }^{2+}$ levels and subsequently also lysosomal ROS levels. Confirming the biological similarity to Salinomycin, Autoquin, too, was found to induce lipid peroxidation, cause lipid membrane permeabilization and was active in a model of human breast cancer stem cells.

Autoquin was identified early as an autophagy inhibitor, however the target candidate obtained by pulldown experiments could not be confirmed. Here, Cell Painting added the essential information of lysosomotropic activity, which then, together with the hypothesis of iron-chelation gathered from the pull-down experiment, lead to the identification of the mechanism of action.

\section{Conclusions}

Pseudo-NPs combine fragments from NPs in ways not found in Nature. These compounds not only occupy new chemical space that is not covered by NPs, they often also have bioactivities and mechanisms of action that are new and unrelated to their parent scaffolds.

Discovering an unknown bioactivity and deconvoluting it to a biological target or mechanism of action can be a challenging endeavour. The Cell Painting assay has proven to be a valuable asset in this process. Due to the unbiased nature of its setup, the assay can identify a wide range of biological activities as long as these manifest themselves in changes of the cell morphology. By comparing the biological profiles of new compounds to those of known and well-described reference compounds, the identification of target clusters or pathways is possible and serves as a starting point for follow-up assays in the target deconvolution procedure. The examples highlighted here have emphasized the potential of the pseudo-NP approach to generate molecules with new and interesting bioactivities. They also demonstrated the relevant input the Cell Painting assay can give at different stages of the target identification process, either directly at the beginning to identify initial bioactivity or at later stages, when activity has already been established by a different technology.

In summary, Cell Painting represents a powerful addition to the available set of target identification strategies.

\section{References}

1. Newman, D. J.; Cragg, G. M. J. Nat. Prod. 2020, 83 (3), 770-803.

https://doi.org/10.1021/acs.jnatprod.9b01285

2. Cragg, G. M.; Newman, D. J. Biochim. Biophys. Acta 2013, 1830 (6), 3670-3695.

https://doi.org/10.1016/i.bbagen.2013.02.008 
3. Wetzel, S.; Bon, R. S.; Kumar, K.; Waldmann, H. Angew. Chem. Int. Ed Engl. 2011, 50 (46), 10800-10826. https://doi.org/10.1002/anie.201007004

4. Over, B.; Wetzel, S.; Grütter, C.; Nakai, Y.; Renner, S.; Rauh, D.; Waldmann, H. Nat. Chem. 2013, 5 (1), $21-$ 28.

https://doi.org/10.1038/nchem.1506

5. Chen, Y.; Garcia de Lomana, M.; Friedrich, N.-O.; Kirchmair, J. J. Chem. Inf. Model. 2018, 58 (8), $1518-1532$. https://doi.org/10.1021/acs.jcim.8b00302

6. Huigens, R. W.; Morrison, K. C.; Hicklin, R. W.; Flood, T. A.; Richter, M. F.; Hergenrother, P. J. Nat. Chem. 2013, 5 (3), 195-202.

https://doi.org/10.1038/nchem.1549

7. Reymond, J.-L.; Awale, M. ACS Chem. Neurosci. 2012, 3 (9), 649-657. https://doi.org/10.1021/cn3000422

8. Erlanson, D. A.; Fesik, S. W.; Hubbard, R. E.; Jahnke, W.; Jhoti, H. Nat. Rev. Drug Discov. 2016, 15 (9), $605-$ 619.

https://doi.org/10.1038/nrd.2016.109

9. Joseph-McCarthy, D.; Campbell, A. J.; Kern, G.; Moustakas, D. J. Chem. Inf. Model. 2014, 54 (3), $693-704$. https://doi.org/10.1021/ci400731w

10. Karageorgis, G.; Foley, D. J.; Laraia, L.; Waldmann, H. Nat. Chem. 2020, 12 (3), 227-235. https://doi.org/10.1038/s41557-019-0411-x

11. Grigalunas, M.; Burhop, A.; Christoforow, A.; Waldmann, H. Curr. Opin. Chem. Biol. 2020, 56, 111-118. https://doi.org/10.1016/i.cbpa.2019.10.005

12. Goto, Y.; Ito, Y.; Kato, Y.; Tsunoda, S.; Suga, H. Chem. Biol. 2014, 21 (6), 766-774. https://doi.org/10.1016/i.chembiol.2014.04.008

13. Ozaki, T.; Yamashita, K.; Goto, Y.; Shimomura, M.; Hayashi, S.; Asamizu, S.; Sugai, Y.; Ikeda, H.; Suga, H.; Onaka, H. Nat. Commun. 2017, 8, 14207. https://doi.org/10.1038/ncomms14207

14. Asai, T.; Tsukada, K.; Ise, S.; Shirata, N.; Hashimoto, M.; Fujii, I.; Gomi, K.; Nakagawara, K.; Kodama, E. N.; Oshima, Y. Nat. Chem. 2015, 7 (9), 737-743.

https://doi.org/10.1038/nchem.2308

15. Kikuchi, H.; Ichinohe, K.; Kida, S.; Murase, S.; Yamada, O.; Oshima, Y. Org. Lett. 2016, 18 (22), 5948-5951. https://doi.org/10.1021/acs.orglett.6b03057

16. Schenone, M.; Dančík, V.; Wagner, B. K.; Clemons, P. A. Nat. Chem. Biol. 2013, 9 (4), 232-240. https://doi.org/10.1038/nchembio.1199

17. Hughes, J.; Rees, S.; Kalindjian, S.; Philpott, K. Br. J. Pharmacol. 2011, 162 (6), 1239-1249. https://doi.org/10.1111/i.1476-5381.2010.01127.x

18. Onyeogaziri, F. C.; Papaneophytou, C. SLAS Discov. Adv. Life Sci. R D 2019, 24 (5), 587-596. https://doi.org/10.1177/2472555219830084

19. Quenching of Fluorescence. In Principles of Fluorescence Spectroscopy; Lakowicz, J. R., Ed.; Springer US: Boston, MA, 2006; pp 277-330. https://doi.org/10.1007/978-0-387-46312-4 8

20. Hall, M. D.; Yasgar, A.; Peryea, T.; Braisted, J. C.; Jadhav, A.; Simeonov, A.; Coussens, N. P. Methods Appl. Fluoresc. 2016, 4 (2), 022001. https://doi.org/10.1088/2050-6120/4/2/022001

21. Maguire, J. J.; Kuc, R. E.; Davenport, A. P. Methods Mol. Biol. Clifton NJ 2012, 897, 31-77. 
https://doi.org/10.1007/978-1-61779-909-9 3

22. Kaur, H.; Lakatos-Karoly, A.; Vogel, R.; Nöll, A.; Tampé, R.; Glaubitz, C. Coupled ATPase-Adenylate Kinase Activity in ABC Transporters. Nat. Commun. 2016, 7, 13864.

https://doi.org/10.1038/ncomms13864

23. Moore, K.; Rees, S. J. Biomol. Screen. 2001, 6 (2), 69-74.

https://doi.org/10.1177/108705710100600202

24. Mazina, O.; Allikalt, A.; Heinloo, A.; Reinart-Okugbeni, R.; Kopanchuk, S.; Rinken, A. Methods Mol. Biol. Clifton NJ 2015, 1272, 65-77. https://doi.org/10.1007/978-1-4939-2336-6 5

25. Gill, S.; Gill, R.; Wicks, D.; Liang, D. Assay Drug Dev. Technol. 2007, 5 (3), 373-380. https://doi.org/10.1089/adt.2006.004

26. Pick, H.; Alves, A. C.; Vogel, H. Chem. Rev. 2018, 118 (18), 8598-8654. https://doi.org/10.1021/acs.chemrev.7b00777

27. Moffat, J. G.; Vincent, F.; Lee, J. A.; Eder, J.; Prunotto, M. Nat. Rev. Drug Discov. 2017, 16 (8), $531-543$. https://doi.org/10.1038/nrd.2017.111

28. Konstantinidis, G.; Sievers, S.; Wu, Y.-W. In Autophagy in Differentiation and Tissue Maintenance: Methods and Protocols; Turksen, K., Ed.; Methods in Molecular Biology; Springer: New York, NY, 2019; pp 187-195. https://doi.org/10.1007/7651_2018_125.

29. Yun, C. W.; Lee, S. H. Int. J. Mol. Sci. 2018, 19 (11), 3466.

https://doi.org/10.3390/ijms19113466

30. Mathew, R.; Karantza-Wadsworth, V.; White, E. Nat. Rev. Cancer 2007, 7 (12), 961-967. https://doi.org/10.1038/nrc2254

31. Peppard, J. V.; Rugg, C.; Smicker, M.; Dureuil, C.; Ronan, B.; Flamand, O.; Durand, L.; Pasquier, B. Curr. Chem. Genomics Transl. Med. 2014, 8 (Suppl-1), 3-15. https://doi.org/10.2174/2213988501408010003

32. Bray, M.-A.; Singh, S.; Han, H.; Davis, C. T.; Borgeson, B.; Hartland, C.; Kost-Alimova, M.; Gustafsdottir, S. M.; Gibson, C. C.; Carpenter, A. E. Nat. Protoc. 2016, 11 (9), 1757-1774. https://doi.org/10.1038/nprot.2016.105

33. Rohban, M. H.; Singh, S.; Wu, X.; Berthet, J. B.; Bray, M.-A.; Shrestha, Y.; Varelas, X.; Boehm, J. S.; Carpenter, A. E. elife 2017, 6, e24060. https://doi.org/10.7554/eLife.24060

34. Hughes, R. E.; Elliott, R. J. R.; Munro, A. F.; Makda, A.; O’Neill, J. R.; Hupp, T.; Carragher, N. O. SLAS Discov. Adv. Sci. Drug Discov. 2020. https://doi.org/10.1177/2472555220917115

35. Melillo, B.; Zoller, J.; Hua, B. K.; Verho, O.; Borghs, J. C.; Nelson, S. D.; Maetani, M.; Wawer, M. J.; Clemons, P. A.; Schreiber, S. L. J. Am. Chem. Soc. 2018, 140 (37), 11784-11790.

https://doi.org/10.1021/jacs.8b07319

36. Gerlach, E. M.; Korkmaz, M. A.; Pavlinov, I.; Gao, Q.; Aldrich, L. N. Systematic Diversity-Oriented Synthesis of Reduced Flavones from Y-Pyrones to Probe Biological Performance Diversity. ACS Chem. Biol. 2019, 14 (7), 1536-1545. https://doi.org/10.1021/acschembio.9b00294

37. CellProfiler https://cellprofiler.org/home (accessed Jul 15, 2020).

38. Christoforow, A.; Wilke, J.; Binici, A.; Pahl, A.; Ostermann, C.; Sievers, S.; Waldmann, H. Angew. Chem. Int. Ed. 2019, 58 (41), 14715-14723. 
https://doi.org/10.1002/anie.201907853

39. Trachootham, D.; Alexandre, J.; Huang, P. Nat. Rev. Drug Discov. 2009, 8 (7), 579-591. https://doi.org/10.1038/nrd2803

40. Foley, D. J.; Zinken, S.; Corkery, D.; Laraia, L.; Pahl, A.; Wu, Y.-W.; Waldmann, H. Angew. Chem. Int. Ed. 2020, 59 (30), 12470-12476.

https://doi.org/10.1002/anie.202000364

41. Laraia, L.; Garivet, G.; Foley, D. J.; Kaiser, N.; Müller, S.; Zinken, S.; Pinkert, T.; Wilke, J.; Corkery, D.; Pahl, A.; Sievers, S.; Janning, P.; Arenz, C.; Wu, Y.; Rodriguez, R.; Waldmann, H. Angew. Chem. Int. Ed. 2020, 59 (14), 5721-5729.

https://doi.org/10.1002/anie.201913712

42. Laraia, L.; Ohsawa, K.; Konstantinidis, G.; Robke, L.; Wu, Y.-W.; Kumar, K.; Waldmann, H. Angew. Chem. Int. Ed Engl. 2017, 56 (8), 2145-2150.

https://doi.org/10.1002/anie.201611670

43. Chen, X.; Wang, Y.; Ma, N.; Tian, J.; Shao, Y.; Zhu, B.; Wong, Y. K.; Liang, Z.; Zou, C.; Wang, J. Signal Transduct. Target. Ther. 2020, 5 (1), 1-13.

https://doi.org/10.1038/s41392-020-0186-y

44. Rix, U.; Superti-Furga, G. Nat. Chem. Biol. 2009, 5 (9), 616-624.

https://doi.org/10.1038/nchembio.216

45. Lu, S.; Sung, T.; Lin, N.; Abraham, R. T.; Jessen, B. A. PLOS ONE 2017, 12 (3), e0173771.

https://doi.org/10.1371/journal.pone.0173771

\section{Authors' Biographies}

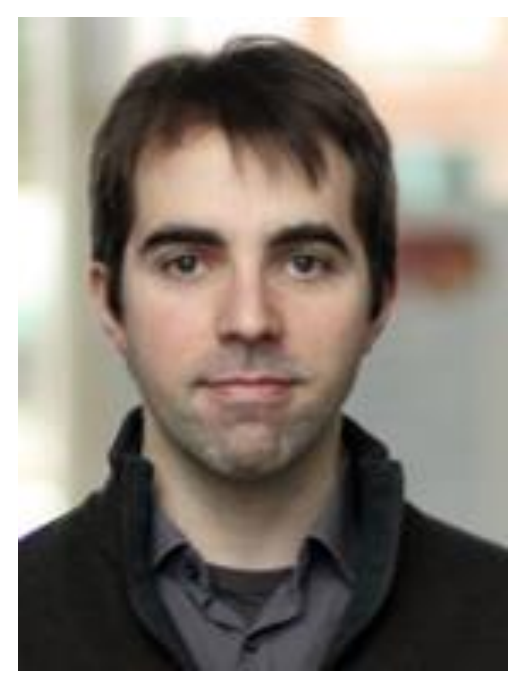

José-Manuel Gally recently completed his PhD in structural bioinformatics and chemoinformatics at the University of Orléans, France. He is currently occupying a Postdoctoral Fellow position at the Max Planck Institute of Molecular Physiology in Dortmund, Germany within the group of Prof. Dr. Dr. h.c. Herbert Waldmann. 


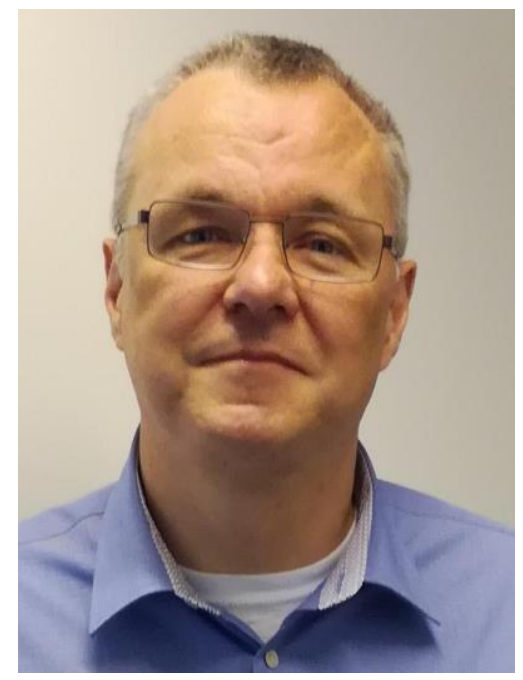

Dr. Axel Pahl has studied organic chemistry at the university of Hannover and has been employed as a Medicinal Chemist in the pharmaceutical industry for more than 10 years. He is now working as a Chemoinformatician / Data Scientist at COMAS, the screening center for the Max Planck Society, and is responsible for the Cell Painting analysis.

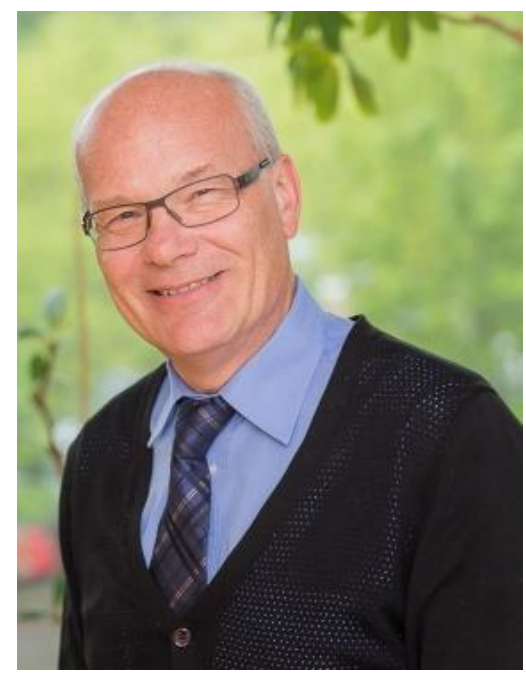

Prof. Dr. h.c. Herbert Waldmann obtained his PhD in organic chemistry in 1985 under the supervision of Horst Kunz. After a postdoctoral period with George Whitesides at Harvard University, he returned to the University of Mainz and completed his habilitation in 1991. He was appointed as Director at MPI Dortmund and professor of Biochemistry at TU Dortmund University in 1999. His research focuses on new principles for the design and syntheses of natural-product-inspired compound classes and their biological evaluation. 